$\underline{\underline{p}}=\mathrm{E}$

\title{
A Working Model for Mobile Charging using Wireless Power Transmission
}

\author{
I. Joe Louis Paul ${ }^{1}$, S. Sasirekha ${ }^{2}$, D. Naveen Kumar $D^{3}$, P.S. Revanth ${ }^{4}$ \\ ${ }^{1,2,3,4}$ Department of IT, SSN College of Engineering, Chennai, India
}

\begin{abstract}
Portable electronic devices are very popular nowadays. Almost all portable devices are battery powered, meaning that eventually, they all must be recharged-using the wired chargers currently being used. As the usage of these portable electronic devices is increasing, the demands for longer battery life are also increasing. These batteries need to be recharged or replaced periodically. It is a hassle to charge or change the battery after a while, especially when there is no power outlet around. Now instead of plugging in a cell phone, Personal Digital Assistant (PDA), digital camera, voice recorder, mp3 player or laptop to recharge it, it could receive its power wirelessly. The technology for Wireless Power Transmission or Wireless Power Transfer or Transmission (WPT) is in the forefront of electronic development. WPT systems are designed to transmit power without using wires more efficient than transmitting it while using wires. There could be large number of applications for wireless power systems. Hence, in this work, a wireless battery charger has been proposed for mobile phone charging which is expected to eliminate all the hassles with today's battery technology. The advantage of this device is that it can wirelessly charge up the batteries which can save time and money in a long run for the general public.
\end{abstract}

Keywords: Wireless Mobile Chargers - Wireless Communication - Cellular Phones - Transmitters - Receivers - Wireless Power Transmission (WPT).

\section{Introduction}

Today, electricity plays a vital role in our modern day life. As we are using many numbers of appliances using electricity, it is quite difficult to live without electricity. Traditionally wires or cables are used to carry the electrical power from one place to another. However, Wireless Power Transmission (WPT) has emerged as the technology in the recent days, where the electrical power is transmitted from one place to another without the use of wires.

The main theme behind WPT is to get rid of the risky usage of the wires at the same time to eliminate the difficulty in organizing the power cords. For an example, the portable electronic devices including mobile phones, tablets, laptops, household robots, drones and etc normally relies upon the battery power. Due to the rapid development and tremendous applications, these portable devices are becoming part of our day to day activities. In addition to that, there is always an increasing demand of smart gadgets to say good bye to wires, making capable of charging without being plugged in. Hence, there is a necessity for finding a new technology to be free of from the clumsy cables or the chargers [1]-[3].

Hence, the researchers at MIT coined the term WiTricity as the part of their project work, where WiTricity is nothing but the Wireless Electricity offering the transmission of electrical power to a remote place without using wires. Basically, WiTricity eliminates the need for having a different charger for each device we use. This is the fundamental advantage we can get it from this technology. It is enough to identify a location where we can put the portable devices which is getting automatically charged. WiTricity ensures that the power hungry devices can charge by their own without the need for plugging in into to power cords using the chargers.
Apart from safety (as there is no need of cables), WiTricity provides more convenient in the sense that there is no need for manual recharging or changing the batteries and seems to be more reliable as the devices will never run out of the battery power. Moreover, WiTricity can make the environment as more ecofriendly as it reduces the use of disposable batteries. Even though WiTricity provides automatic wireless charging, mainly requires short distances to charge. Hence, WiTricity is still under development where lots of research works are going on to improve its potential applications to even charge the larger vehicles or equipments and also being operated over a longer distances.

Hence, this work aims to propose a novel method of using WiTricity to charge the mobile phones without the use of wired chargers. The significance of this work is to make an efficient power transfer of low voltage over a shorter distance. The proposed work promises that the mobile users can carry their phones anywhere even if the place is devoid of charging facilities [4]-[6].

\section{Related Works}

Whenever we use the devices in our day to day activities and whatever may be the devices we use, it becomes essential to recharge the devices regularly with the help of wired chargers. But, there may be a situation that there may be no power supply due to some natural hazards may include cyclone, earth quake and etc. In that case, is there any possibility to transfer the power from one mobile phone to another mobile phone wirelessly? Keerthana and Pragadeshwar addressed this issue in their work by eliminating the need of physical power supply to the portable devices where the easy transfer of power from one mobile phone to another mobile phone can be done using inductive coupling [7]. This might be very useful in emergency conditions. 
There may be certain places where the wired systems can be unreachable or impossible. Hence, without using wires, there should be way for finding the effective methods to transfer the power between two pints is the need of the hour. Sharma et al addressed this issue in their work [8]. The power can be transferred by any one of the following three methods namely inductive coupling (for short-range), resonant induction (for midrange) and electromagnetic wave transmission (for long-range). The objective of this work was to charge a low power device using inductive coupling by quickly and efficiently [9].

Likewise, it may not be always possible to carry the mobile phone chargers wherever we go. It is quite hard to carry the mobile phone chargers at all times at all places. Vithyaa and Marthandan attempted to address the implementation issues of WPT usage for mobile phones and vehicles in their work [10].

Zaman et al emphasized the need for designing a simplified model to transfer the electrical power from one mobile to another mobile phone using the series-series technology for charging inductively [11]. It is inferred that more development is required for designing a low cost, more efficient, simple WPT which can be compatible with any phone model.

Electric vehicles are getting more attention today rather than traditional counterparts (which rely on natural resources) due to its nature of environment friendly. On et al presented an experimental method of WPT for charging the electric vehicles using inductive coupling method [12].

In continuation to that, Sultana et al proposed the design of WPT based charging system for electric vehicles by placing the WPT circuitry into the electrical vehicles [13]. This circuitry was activated when the electrical vehicles reached the charging area mainly to overcome the battery related issues in electrical vehicles. The pros and cons of each WPT technologies, the research objectives of WPT such as improving the transmission efficiency and the distance, current state-of-art technologies of WPT, thee open research challenges of WPT are discusses by Mou in his work. Even the possibility of charging multiple devices using WPT in the near future is also presented in [14].

\section{System Architecture}

The proposed system architecture consists of two sections namely transmitter and receiver sections. Table 1 illustrates the hardware requirements for our proposed work.

Table 1: Hardware Requirements

\begin{tabular}{|c|l|l|}
\hline Sl. No. & \multicolumn{1}{|c|}{ Hardware Name } & \multicolumn{1}{c|}{ Specification } \\
\hline 1 & Step Down Transformer & $230 \mathrm{~V}-12 \mathrm{~V}$ \\
\hline 2 & Diode(4 Nos) & $1 \mathrm{~N} 4007$ \\
\hline 3 & Voltage regulator & 7812 \\
\hline 4 & PIC Board & $12 \mathrm{~V}$ Input \\
\hline 5 & PIC Microcontroller & PIC16F887 \\
\hline 6 & Relay Switch & $12 \mathrm{~V}$ \\
\hline 7 & Wireless Charging Module & XKT-510 and T3168 \\
\hline 8 & Capacitor & $220 \mathrm{uF}, 6.8 \mathrm{nF}, 100 \mathrm{uF}$ \\
\hline 9 & Resistor & $1 \mathrm{~K}, 10 \mathrm{~K}, 98 \mathrm{Ohm}$ \\
\hline
\end{tabular}

\subsection{Transmitter Section}

Figure 1 shows the transmitter section which starts with an input AC supply of $230 \mathrm{~V}$. Then step down transformer is used for reducing the input voltage. Actually, step down transformer converts the $230 \mathrm{~V}$ AC supply into $12 \mathrm{~V}$ AC supply. Then, the bridge rectifier converts the AC supply to DC supply. Reason for using bridge rectifier is that we need only DC supply for charging the mobile. Next, the voltage regulator is used for reducing the repulsion in DC supply. Finally, the DC supply is transferred to the transmitter coil. The wireless power transmitting coil would create an alternating magnetic field in the coil due to induction, to transmit energy.

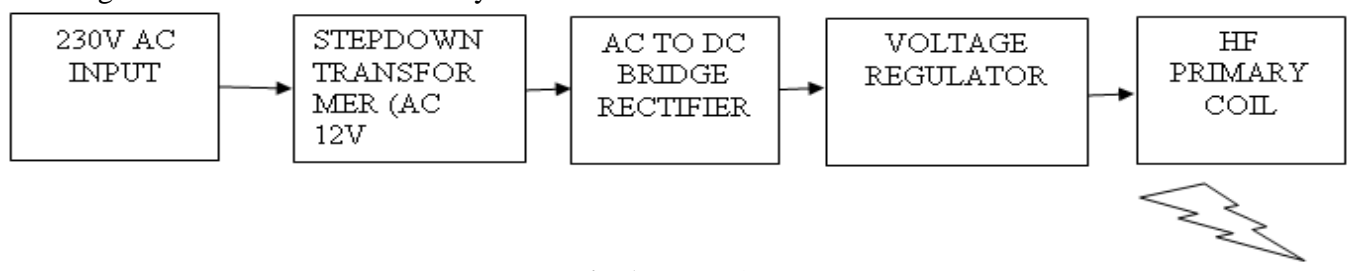

Fig. 1: Transmitter

\subsection{Receiver Section}

Receiver section consists of receiver coil. The AC current from the receiver coil is admitted for rectification and filters to convert $\mathrm{AC}$ into DC. Then, the DC supply is admitted to the DC regulator (7812) to change the DC supply to $12 \mathrm{~V}$ DC. Next, the $12 \mathrm{~V}$ DC is applied as an input to the PIC 16F877A.Then the output is connected to the mobile adapter. Since we are obtaining very low DC supply, a mobile adapter is used. Using that adapter, mobile phone is admitted to the charging as shown in Fig. 2.

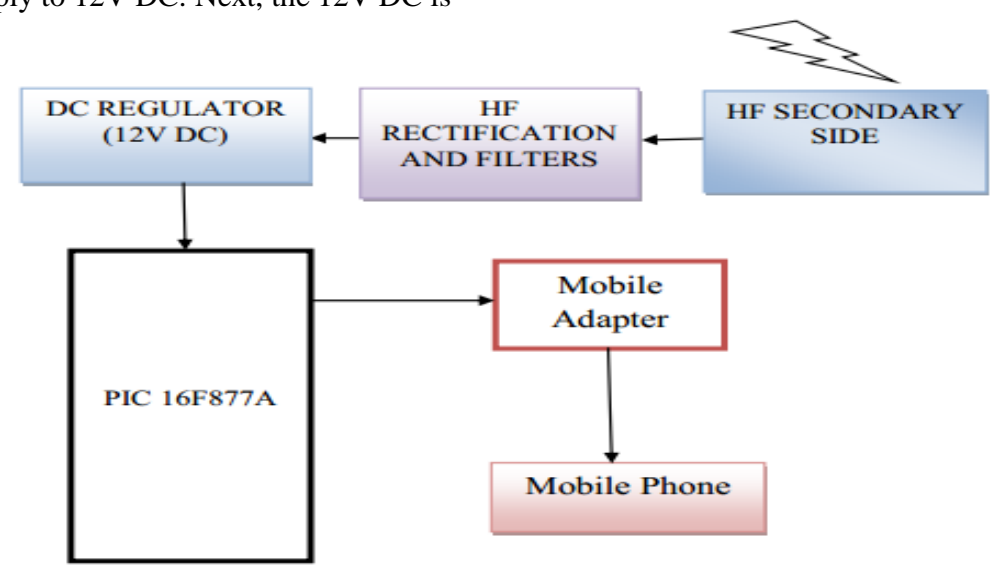

\section{Software Implementation}

\subsection{ISIS Simulation}

Fig. 2: Receiver

In ISIS simulation, the transmitter circuit is used to check the connections. Here, an input voltage of $12 \mathrm{~V}$ is given, where the output value of $4.7 \mathrm{~V}$ is obtained as shown in Fig. 3. From this, we 
have come to the conclusion that, no errors in the circuit is obtained.

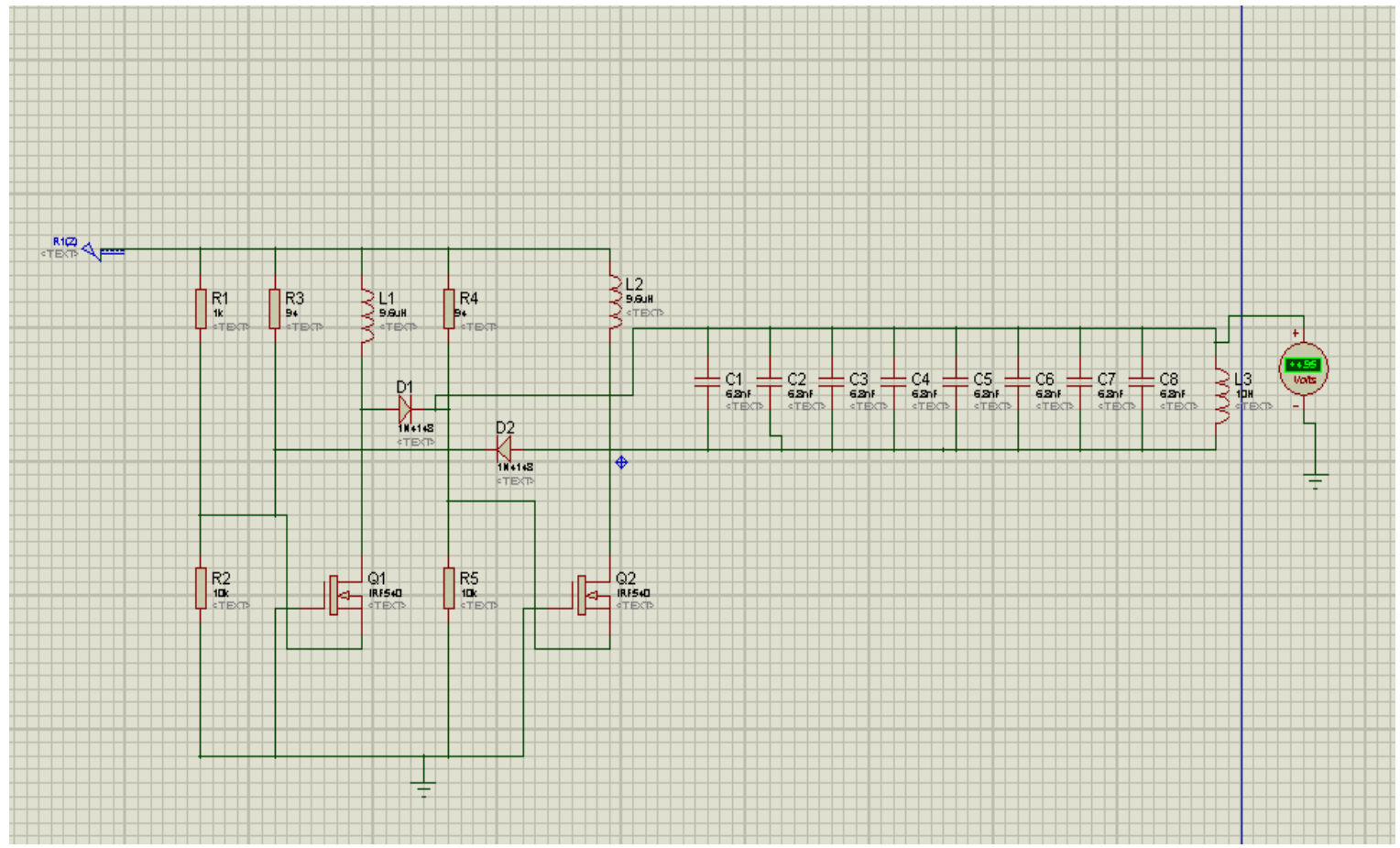

Fig. 3: ISIS Simulation-Transmitter

In ISIS simulation, the receiver circuit is also done to check the connections. Here, the input voltage of $5 \mathrm{~V}$ is given, where the output value of $2 \mathrm{~V}$ has been obtained as shown Fig 4. From this, it is concluded that, no errors in the circuit is found.

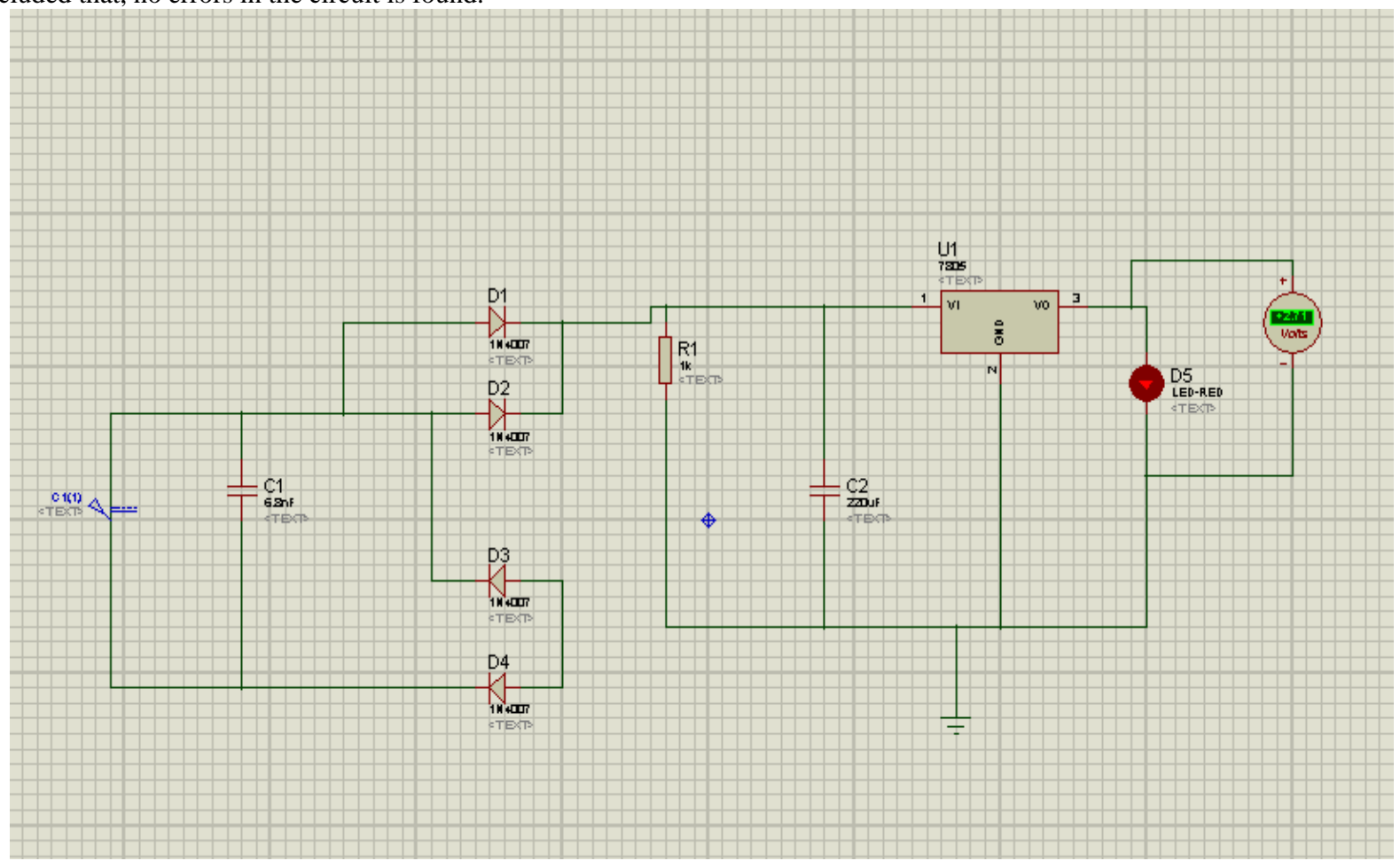

Fig. 4: ISIS Simulation-Receiver 


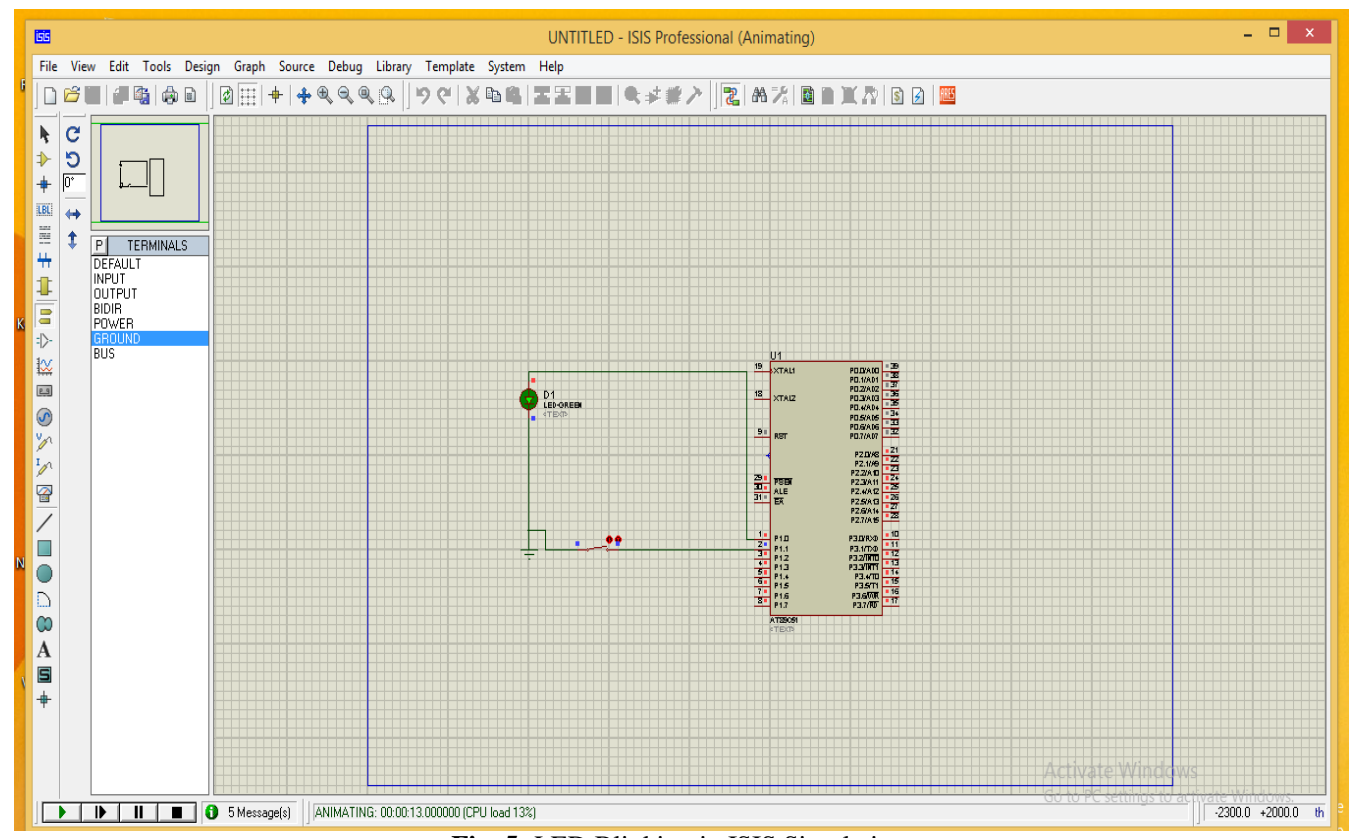

Fig. 5: LED Blinking in ISIS Simulation

PIC program is loaded into the PIC microcontroller embedded in the PIC board by using PIC KIT 3. As shown in Fig 5, LED is blinking by running the above program in ISIS Simulation.

\section{Hardware Implementation}

Blinking of the LED light is found by loading the program into the PIC microcontroller and connecting LED light into one of its port to get the output as shown in Fig. 6.

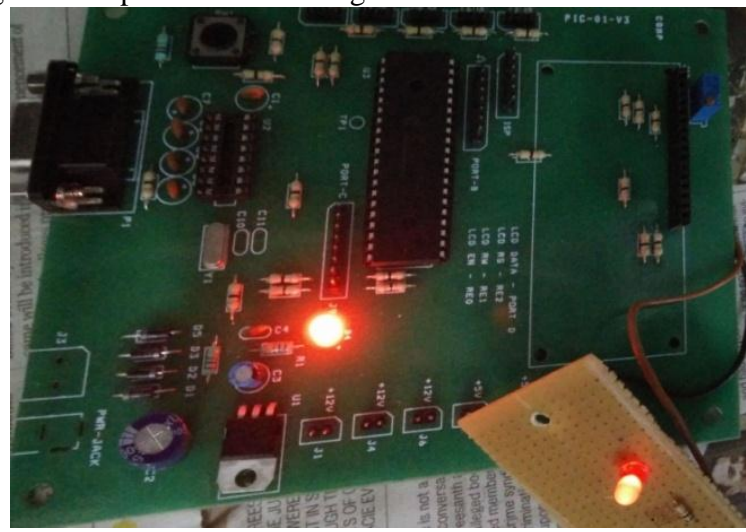

Fig. 6: LED Blinking in PIC Board

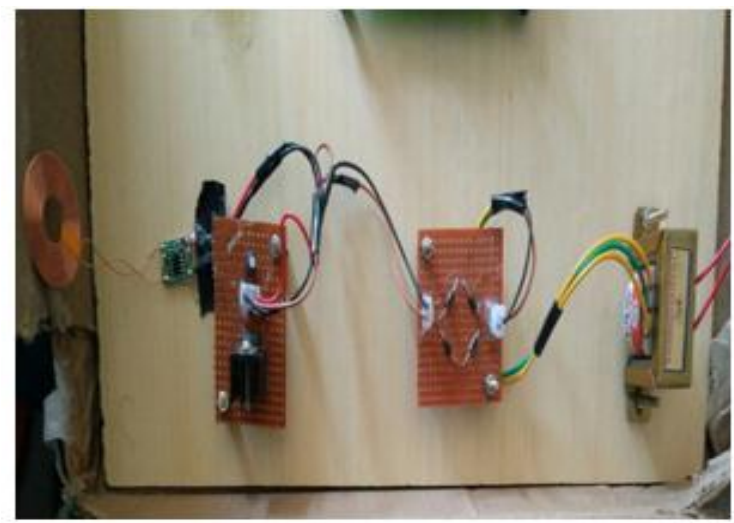

Fig. 7: Transmitter Section

After finishing the LED blinking, the transmitter section is constructed. Fig. 7 shows the detailed view of transmitter. Here, the components like transformer, bridge rectifier, regulator and a transmitter coil are used.

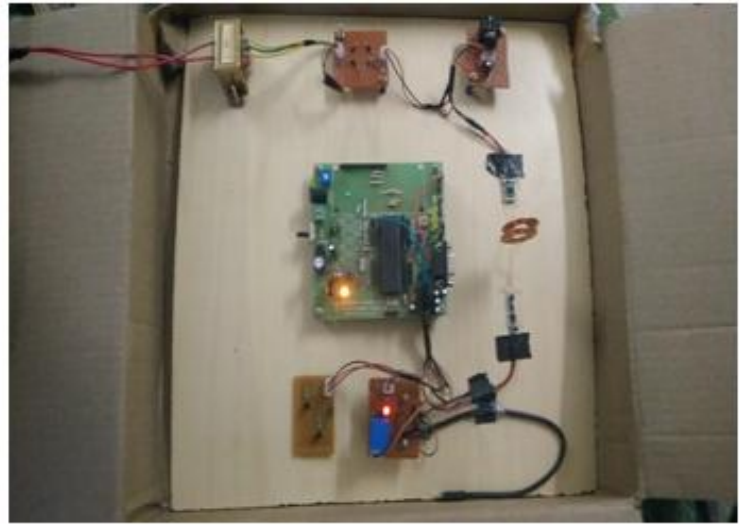

Fig. 8: Receiver Section

After completing the transmitter section, the receiver section is added which in turn connected to the PIC board and tried to blink the LED. Since, the constant voltage is not obtained; the relay switch is used to charge the phone.

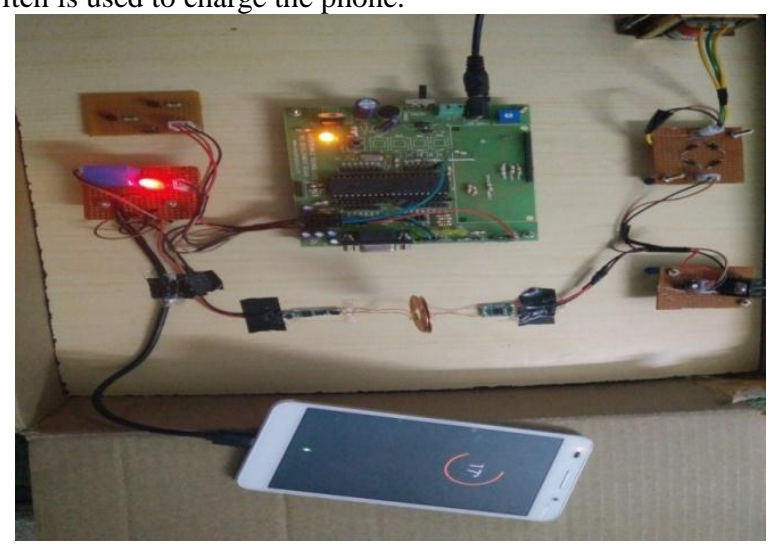

Fig. 9: Charging Phone using WPT

From Fig. 9, it is observed that the mobile device is getting charged. But the problem with this model is that it produces low output voltage. Since the output voltage is low, the charging rate of the mobile device is less. Increasing the dimension of the coil and bringing them in close proximity would form a sufficing solution to the above. Instead of using a single pair of inductor coils, multiple pairs connected in parallel could be used to enhance the production of output voltage. 


\section{Conclusion and Future Work}

Wireless battery charging has many advantages in terms of convenience because users simply need to place the device requiring power onto a mat or other surface to allow the wireless charging to take place. We believe that our contribution in this work will successfully benefit society in terms of convenience, reduced wear of plugs and sockets, and application in medical environments. Reduced efficiency is one of the key challenges in wireless battery charging system due to resistive losses on the coil, stray coupling and etc.

\section{References}

[1] Xiao Lu, Niyato, D, Ping Wang, Dong In Kim, Zhu Han, "Wireless charger networking for mobile devices: fundamentals, standards, and applications", IEEE Wireless Communications, Vol. 22, No. 2 , pp. 126 - 135, April 2015.

[2] Siqi Li , Mi, C.C., "Wireless Power Transfer for Electric Vehicle Applications", IEEE Journal of Emerging and Selected Topics in Power Electronics, Vol. 3, No.1, pp. 4-17, April 2014.

[3] Olvitz, L, Vinko D, Svedek T, "Wireless power transfer for mobile phone charging device", Proc. $35^{\text {th }}$ IEEE International Convention MIPRO, pp. 141 - 145, May 2012.

[4] Tsunekawa K, "A feasibility study of wireless power transmission system by using two independent coupled electric fields", IEEE MTT-S International Microwave Workshop Series on Innovative Wireless Power Transmission: Technologies, Systems, and Applications (IMWS), pp. 141 - 144, May 2011

[5] Shubham Chaturvedi, Er. Sudhanshu Tripathi, "Modelling \& Analysis of High-Power, High-Frequency Wireless Power Transmission", International Research Journal of Engineering and Technology (IRJET), Vol. 04, No. 05, pp. 2732- 2737, May 2017.

[6] Md. Saifuddin, Tushar Saha, Monirul Islam, Md. Mujammel Hossain Akhand, Ebad Zahir, "Wireless Power Transmission Compare and Contrast with the Form of Resonance Frequency, Mutual Inductance and Solar Energy, International Journal of Engineering Research and Application, Vol. 4, No. 10 (Version 6), pp.66-96, October 2014.

[7] Keerthana.R, Pragadeshwar.A.M, "Wireless Mobile to Mobile Charge Transfer Using WPT Technology", International Journal of Advanced Research in Computer Science Engineering and Information Technology, Vol. 4, No. 3, Special Issue: 3, pp. 984 990, April, 2016

[8] Jitendra Sharma, Kavita Jain, Muralidhar Jangid, Rakesh Prajapat, Shubham Sharma, Pradeep Yadav, Nikhil Gupta, "International Journal of Engineering and Management Research", Vol. 6, No. 2, pp. 248-251, March-April 2016.

[9] Mohamed A. Hassan, A.Elzawawi, "Wireless Power Transfer through Inductive Coupling", Recent Advances in Circuits, pp. 115 118, 2015.

[10] Vithyaa. M, Marthandan. R, "Wireless Power Transmission for Mobile and Vehicle", IOSR Journal of Electronics and Communication Engineering (IOSR-JECE), Vol. 9, No. 1, Ver. VI, pp. 50-52, Feb. 2014

[11] Hasan U. Zaman, Touhidul Islam, Kazi Shanjidul Hasan, Rifat Kamal Antora, "Mobile phone to mobile phone wireless power transfer", Proc. International Conference on Advances in Electrical Engineering (ICAEE), pp. 206-209, Dec 2015.

[12] ChanKam On, Tony Tsang, "A Experiment Method of Wireless Power Transfer for Charging Devices", American Journal of Engineering Research (AJER), Vol. 5, No. 11, pp, 110-123, 2016

[13] Gousia Sultana, Deepak.T.R, Pratiksha Bhushan, Mohammed Azeem , Swathi.G.N, "Design and Implementation of Wireless Power Transfer Charging System on Miniature Model", SSRG International Journal of Electrical and Electronics Engineering (SSRG-IJEEE), Vol. 3, No. 4, March 2016.

[14] Xiaolin Mou, Hongjian Sun, "Wireless Power Transfer: Survey and Roadmap", Proc. IEEE $81^{\text {st }}$ Vehicular Technology Conference (VTC Spring), arXiv:1502.04727v1 [cs.IT], 2015. 